1 Zucchelli P, Zuccala A. Ischemic renal failure. Contrib Nephrol 1996;119:59-66.

2 Donohue $\mathrm{P}$, de Takats D, Bishop N, et al. A four-year audit of interventional treatment for atheromatous renal artery of interventional treatment for atheroma

3 Mailloux LU, Napolitano B, Bellucci AG, Vernace M Wilkes BM, Massey RT. Renal vascular disease causing Wilkes BM, Massey RT. Renal vascular disease causing end-stage renal failure: incidence, clinical correlates and 1994;24:622-9.
4 Missouris CG, Buckenham T, Vallance PJT, MacGregor GA. Renal artery stenosis masquerading as congestive cardiac failure. Lancet 1993;341:1521-2.

5 Olin JW, Melia M, Young JR, Graor RA, Risius B Prevalence of atherosclerotic renal artery stenosis in patient with atherosclerosis elsewhere. Am f Med 1990;88:146N $151 \mathrm{~N}$.

\title{
Pleural effusion and a pelvic mass
}

\author{
D Agranoff, D May, C Jameson, G K Knowles
}

A 56-year-old woman presented with a nine-month history of lassitude, weight loss, an unproductive cough and increasing breathlessness. There was no complaint of chest pain or haemoptysis and she had never smoked. Examination revealed signs of a massive right pleural effusion, confirmed on chest X-ray (figure 1), and a large pelvic mass. There was no clinically detectable ascites, hepatosplenomegaly or lymphadenopathy. Full blood count, erythrocyte sedimentation rate, urea and electrolytes, liver function tests and blood calcium were all normal.

Approximately four litres of yellow fluid were removed by pleural drainage and multiple pleural biopsies were obtained. At laparotomy, a large mass was discovered in the right ovary (figure 2). The uterus contained multiple fibromyomata but no peritoneal nodules were present and there was no ascites.

Kingston Hospital, Galsworthy Road, Kingston upon Thames, Surrey KT2 7QB, UK Department of Medicine

D Agranoff

G K Knowles

Department of

Obstetrics and

Gynaecology

D May

Department of

Histopathology

C Jameson

Correspondence to $\mathrm{Dr}$

Knowles

Accepted 19 November 1997

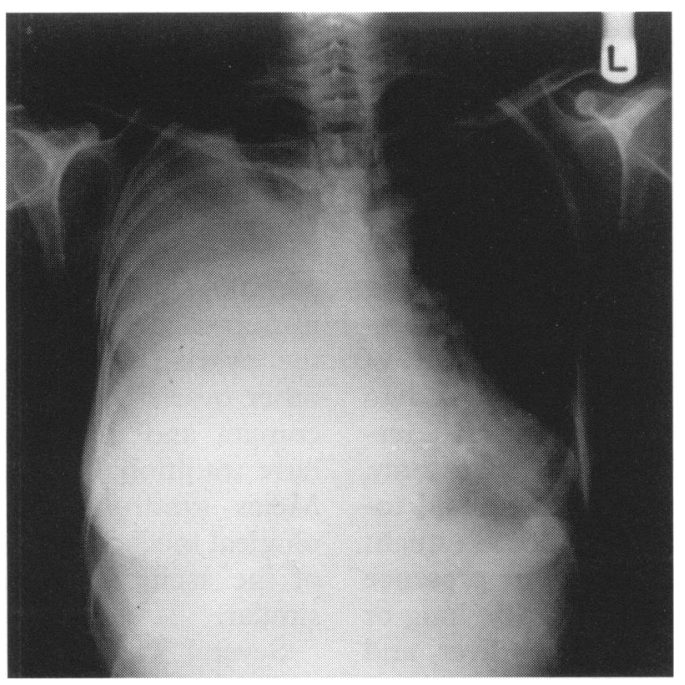

Figure 1 Chest X-ray showing right pleural effusion

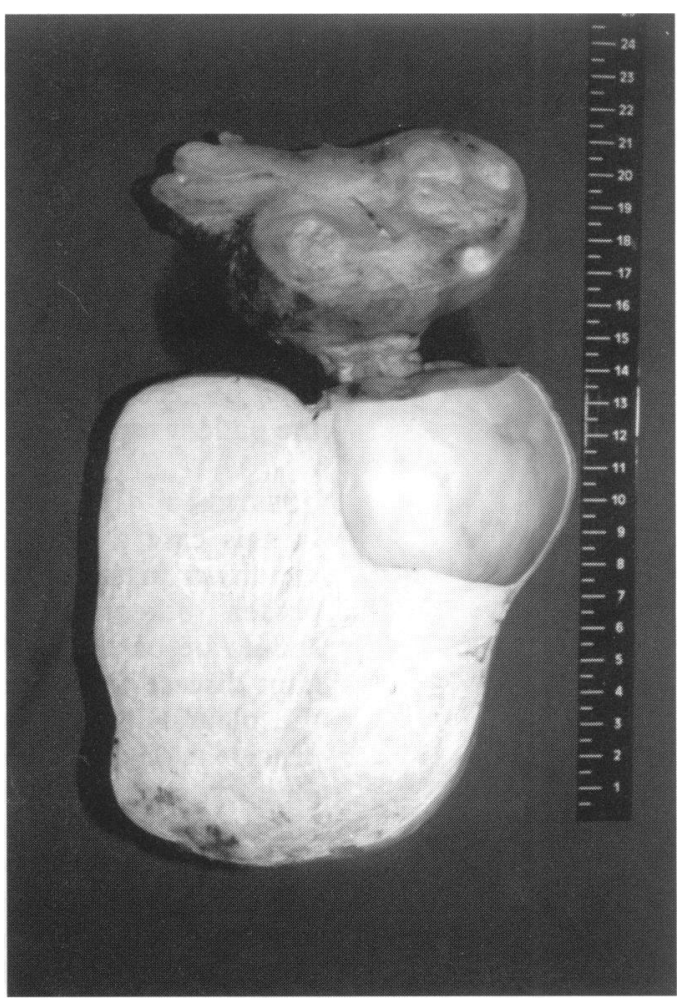

Figure 2 Right ovarian tumour attached to uterus containing multiple fibromyomata

\section{Questions}

1 What is the most probable diagnosis?

2 What additional diagnoses should be considered?

3 What is the correct management in this situation? 


\section{Answers}

QUESTION 1

Ovarian adenocarcinoma with metastatic disease.

QUESTION 2

Intrathoracic malignancy, eg, bronchial carcinoma, mesothelioma or lymphoma with secondary pelvic disease, could explain the clinical and radiographic findings. Other causes of a pleural effusion with a pelvic mass which would need to be considered include tuberculosis and a right subphrenic abscess with an overlying effusion secondary to a pelvic infective lesion. Meigs syndrome, which is characterised by the association of benign ovarian neoplasia, pleural effusion and ascites, should also be included in the differential diagnosis. ${ }^{1}$ In our patient, the ovarian mass proved to be a benign fibrothecoma consisting microscopically of dense bundles of spindle-shaped cells intermingled with larger cells of stromal origin. The pleural fluid cytology and histology of pleural biopsies showed no evidence of neoplasia but would not on their own exclude malignancy.

The present case represents an atypical example of Meigs syndrome in view of the absence of ascites. We know of only one other case described in the literature in which ascites was not a prominent feature but in which blood-stained bilateral pleural effusions occurred in association with a granulosa-cell tumour and which dissipated spontaneously following its removal. ${ }^{2}$ As a rule, the pleural effusion resolves in Meigs syndrome and does not recur once the ovarian tumour has been removed.

At no stage during the initial investigations, at surgery or during follow-up, have we identified any evidence of malignancy or other significant pathology. Three years on there has been no recurrence of the pleural effusion and the patient remains in good health.

\section{QUESTION 3}

In this case a rare and unexpectedly benign condition masqueraded as malignancy. Until proven otherwise, the initial management should be based on the assumption of malignant disease. In the first instance, drainage of the pleural effusion should be performed to provide symptomatic relief and to alleviate respiratory embarrassment prior to laparotomy. Ultrasound examination and/or computed tomography scan of thorax and abdomen might assist by confirming or excluding the presence of additional disease in the underlying lung or in organs below the diaphragm, eg, liver, and by delineating the extent of the ovarian lesion. Serum markers of malignancy might be of value and thoracoscopy would allow direct inspection of the pleural surfaces for deposits.

The effusion is likely to recur with malignant pleural involvement and chemical pleurodesis should be considered. In our case, tetracycline pleurodesis was performed presumptively. Histological examination of the ovarian mass is essential and laparotomy with complete removal of the tumour was carried out. This also

\section{Learning points}

- a large pleural effusion and a pelvic mass may have a benign cause

- the absence of ascites should not preclude Meigs syndrome

afforded the opportunity to examine the peritoneum and other intra-abdominal organs for evidence of malignant deposits.

In spite of the benign findings, regular follow-up would be essential as the diagnosis of a benign syndrome is a retrospective one based essentially on exclusion

\section{Discussion}

The occurrence of a pleural effusion (usually on the right) together with ascites and an ovarian fibroma or fibrothecoma was first described as a well defined clinical syndrome by Meigs and Cass in $1937 .{ }^{1}$ Characteristically, the effusion resolves and does not recur if the ovarian tumour is removed.

Meigs syndrome is a rare condition. Fibromas represent about $4 \%$ of ovarian tumours and Meigs syndrome occurs in only $1-2 \%$ of these cases. Its principal clinical interest lies in the ease with which it may be confused with malignant disease. The distinction may be rendered all the more difficult pre-operatively by the occasional discovery of an elevated serum CA-125, a well recognised marker of ovarian malignancy but which, in Meigs syndrome, appears to be a consequence of non-specific serous membrane stimulation. ${ }^{34}$ In other cases, recurrent pleural effusion may be the presenting feature, the underlying ovarian tumour only coming to light after exclusion of intrathoracic malignancy, cardiac failure, tuberculosis, liver and kidney disease or connective tissue disease. ${ }^{5}$

Since its original description, a number of variants of the syndrome have been reported with differing underlying gynaecological pathologies. These include uterine fibromyomata, ${ }^{6}$ degenerative ovarian changes, ${ }^{7}$ the ovarian hyperstimulation syndrome, and other ovarian stromal tumours including thecomata and granulosa cell tumours. ${ }^{2}$ These have traditionally been referred to as pseudoMeigs syndrome, although the pathophysiological mechanisms involved in the formation of the ascitic and pleural fluid are probably similar.

Several hypotheses have been advanced to explain the formation of the ascitic fluid, including mechanical irritation of the peritoneum by a heavy, mobile tumour, venous and lymphatic congestion of the tumour itself or even the production of vasoactive substances. It is generally supposed that the hydrothorax arises secondary to passage of ascitic fluid into the pleural space via the diaphragm, either through congenital defects which tend to be commoner on the right, or via diaphragmatic lymphatics. It is certainly far commoner to find 
an ovarian fibroma associated with ascites in the absence of a pleural effusion than a pleural effusion in the absence of ascites. In our case, it is certainly possible that drainage of the pleural effusion prior to surgery could have succeeded in drawing off a small ascitic collection by virtue of the putative trans-diaphragmatic continuity between the pleural and peritoneal spaces.

Our management in this instance with formal intercostal drainage and tetracycline

1 Meigs JV, Cass JW. Fibroma of the ovary with ascites and hydrothorax. Am 7 Obstet Gynecol 1937;33:249-67.

2 Martin F, Brouche S. Haidar A. Demons-Meigs syndrome. Report of a case with ovarian tumour of the granulosa. Rev Report of a case with ovar Clin 1990;123-4.

3 Jones OW, Surwit EA. Meigs syndrome and elevated CA125. Obstet Gynecol 1989;73:520-1.

4 Lin JY, Angel C. Meigs syndrome with elevated CA125. Obstet Gynecol 1992; 80:563-6. pleurodesis represented a pragmatic and preoperatively desirable approach to an apparently malignant condition which proved unexpectedly benign at pelvic surgery.

\section{Final diagnosis}

Atypical Meigs syndrome.

Keywords: Meigs syndrome; ovarian tumour

5 Mohaupt M, Overlack A, Vetter H. Dyspnoea at rest (clinical conference). Praxis 1989;78:1003-6.

6 Hander EC, Fran RE, Snashell PD. Atypical Meigs 6 Hander EC, Fran RE, Snashell

7 Teshima T, Iseki K, Nomiyama M, Hirakawa T, Fujishima M A variant of Meigs syndrome without ovarian neoplasia. Respir Med 1989;83:363-5.

\title{
Omental cake - cause?
}

\author{
A I Rhodes, R Joarder, A Al-Kutoubi
}

A 45-year-old women presented to her general practitioner with abdominal and bilateral loin pain. She had recently developed diarrhoea and on examination was found to have a pelvic mass. Initial investigations with ultrasound and a barium enema were followed by a computed tomography (CT) scan of the abdomen and pelvis. Two slices from the CT scan are shown in figures 1 and 2.

Department of Diagnostic Radiology,

St Mary's Hospital, Praed Street, London W2 1NY, UK

AI Rhodes

R Joarder

A Al-Kutoubi

Correspondence to $\mathrm{Dr} \mathrm{AI}$ Rhodes, 12 Camel Grove, Kingston-Upon-Thames, Surrey KT2 5GR, UK

Accepted 24 October 1997
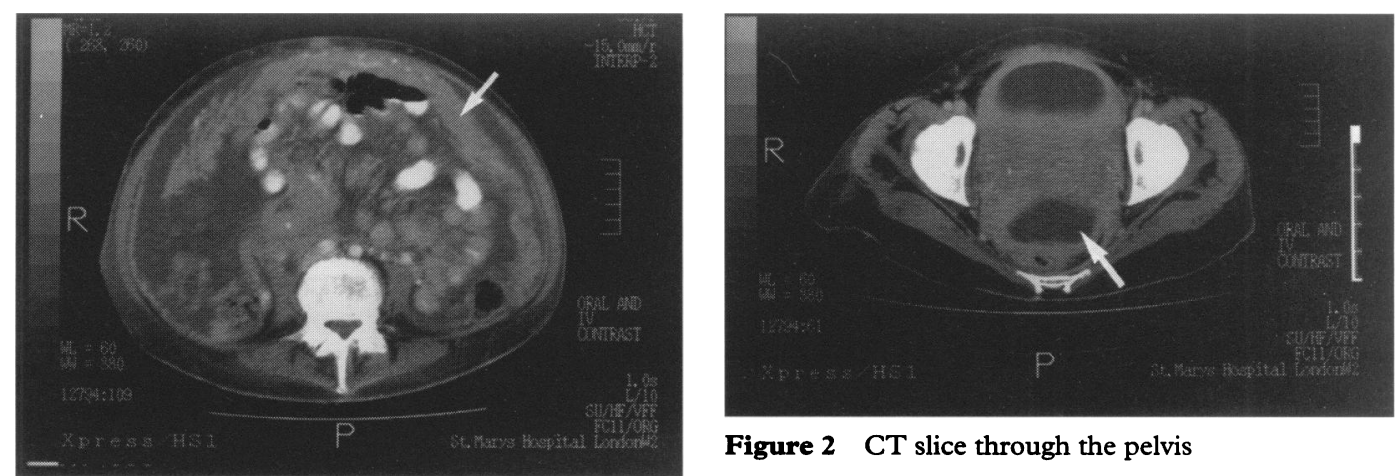

Figure 2 CT slice through the pelvis

Figure 1 CT slice through the abdomen, taken following ingestion of oral contrast and dynamically enhanced with intravenous contrast

\section{Questions}

1 What are the CT findings?

2 What is the differential diagnosis of these appearances? 